\title{
Suppression of Zeeman Gradients by Nuclear Polarization in Double Quantum Dots
}

\author{
S. M. Frolov, ${ }^{1}$ J. Danon, ${ }^{2}$ S. Nadj-Perge,${ }^{1}$ K. Zuo, ${ }^{1}$ J. W. W. van Tilburg, ${ }^{1}$ V. S. Pribiag, ${ }^{1}$ \\ J. W. G. van den Berg, ${ }^{1}$ E. P. A. M. Bakkers, ${ }^{1,3}$ and L. P. Kouwenhoven ${ }^{1}$ \\ ${ }^{1}$ Kavli Institute of Nanoscience, Delft University of Technology, 2600 GA Delft, The Netherlands \\ ${ }^{2}$ Dahlem Center for Complex Quantum Systems, Freie Universität Berlin, 14195 Berlin, Germany \\ ${ }^{3}$ Department of Applied Physics, Eindhoven University of Technology, $5600 \mathrm{MB}$ Eindhoven, The Netherlands
}

(Received 7 September 2012; published 5 December 2012)

\begin{abstract}
We use electric dipole spin resonance to measure dynamic nuclear polarization in InAs nanowire quantum dots. The resonance shifts in frequency when the system transitions between metastable high and low current states, indicating the presence of nuclear polarization. We propose that the low and the high current states correspond to different total Zeeman energy gradients between the two quantum dots. In the low current state, dynamic nuclear polarization efficiently compensates the Zeeman gradient due to the $g$-factor mismatch, resulting in a suppressed total Zeeman gradient. We present a theoretical model of electron-nuclear feedback that demonstrates a fixed point in nuclear polarization for nearly equal Zeeman splittings in the two dots and predicts a narrowed hyperfine gradient distribution.
\end{abstract}

PACS numbers: 73.63.Kv, 72.25.-b

Hyperfine interaction couples electron spin to nuclear spins enclosed by the electron's wave function. In the context of spin qubits in III-V semiconductors, the most prominent effect of this interaction is that fluctuating nuclear spins cause electron spin dephasing [1-3]. Interestingly, ideas for suppressing nuclear spin fluctuations also rely on the same hyperfine interaction, since electron spin transport can lead to dynamical nuclear polarization (DNP) in quantum dots [4]. An experimental manifestation of DNP is a hysteretic current in the spin blockade regime [5-8]. Sometimes, including in the present work, the hysteresis could be extended to high magnetic fields, suggestive of a large degree of nuclear polarization $[9,10]$.

In this report, we study hysteretic spin blockade in InAs nanowire quantum dots using electric dipole spin resonance (EDSR) spectroscopy [11-13]. Surprisingly, the degree of polarization deduced from EDSR does not exceed a few milliTesla, much smaller than the hysteresis range. We explain this apparent contradiction by nuclear fields compensating the natural Zeeman energy difference between the two quantum dots caused by the mismatch of their $g$ factors [14]. In this case one of the double dot states $\left(T_{0}\right)$ is blocked leading to a reduced current. We support this idea by analytical and numerical calculations of spin blockade transport in the presence of hyperfine and spinorbit interactions.

From our model we deduce a narrowing of the hyperfine gradient distribution to a few percent of the unpumped distribution width. This finding is especially relevant for two-electron singlet-triplet qubits, where the hyperfine gradient is the source of dephasing [15]. We predict an order of magnitude enhancement in the coherence time due to gradient suppression induced by spin blockade transport. This is an alternative route to $T_{2}$ enhancement compared with nuclear spin pumping by pulsing the double dot through an $S$ - $T$ transition [16-18].

We present data from two devices that were studied in two previous publications $[3,19]$. InAs nanowires, $40-80 \mathrm{~nm}$ in diameter, are deposited on top of five narrow bottom gates which are then used to define few electron double quantum dots. The nanowires are contacted by Ti/Al leads to measure electron transport through the system. All measurements are performed at $T=250-300 \mathrm{mK}$ in ${ }^{3} \mathrm{He}$ refrigerators.

We tune the double dots to the so-called strong coupled spin blockade regime, in which the current exhibits a dip rather than a peak at zero magnetic field due to an interplay between tunnel coupling, hyperfine, and spin-orbit interactions $[10,19]$. Figure 1(a) shows the double dot current in the vicinity of a spin-blocked charge degeneracy point, which appears as a double-triangle shape when the left and right gates are swept at a finite dc voltage bias $V_{\mathrm{dc}}$ across the double dot. Inside the triangles, one can see sudden transitions between a low and a high current state. The difference in current between the two states exceeds, for some settings, an order of magnitude (1-2 pA vs $10-20 \mathrm{pA})$. This switching is only visible within the boundaries of the triangles. The boundaries themselves remain fixed in gate voltage. This indicates that the jumps in current do not originate from charge switches in the vicinity of the double dot.

The appearance of the two current states is strongly influenced by an applied magnetic field $B_{\text {ext }}$ [Fig. 1(b)]. At zero field we always observe a stable low current because we are in a zero-field dip characteristic of all strongly coupled quantum dots $[5,19,20]$. Usually this dip is hundreds of $\mathrm{mT}$ wide with current increasing smoothly with the field. However, in the regime where we observed the switching, the current drops when the field increases 

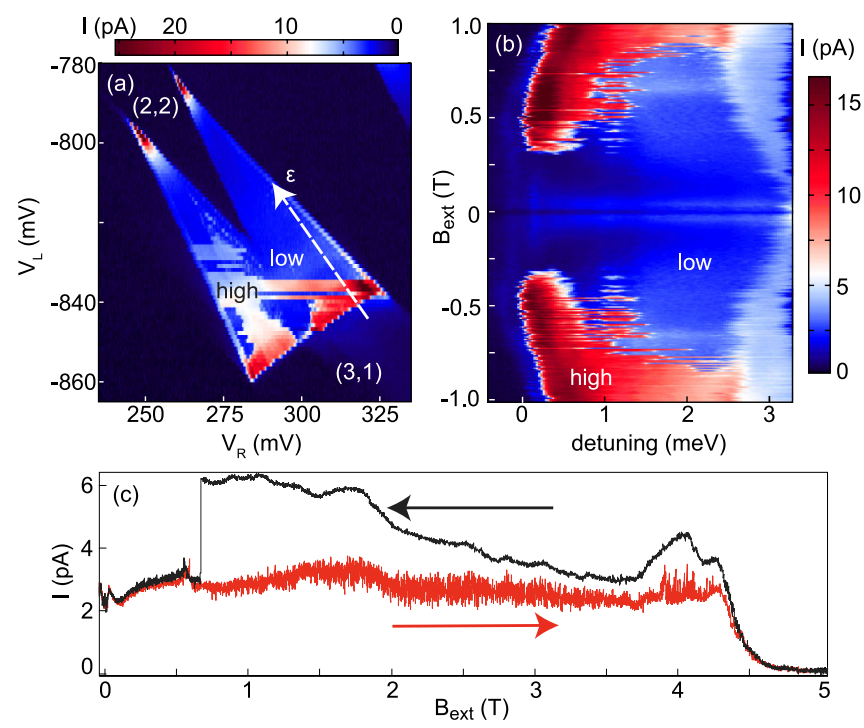

FIG. 1 (color online). (a) Charge stability diagram near the $(3,1) \rightarrow(2,2)$ transition $\left(V_{\mathrm{dc}}=7 \mathrm{mV}, B_{\text {ext }}=0.79 \mathrm{~T}\right)$. Low and high current states are labeled, the dashed arrow indicates the detuning axis $\varepsilon$. (b) Dependence of the leakage current on the magnetic field and detuning. The detuning is swept along the line in panel (a), and the field is stepped after each sweep. For (a) and (b) the sweep direction is from left to right. (c) The magnetic field retrace at fixed detuning $\varepsilon \approx 3.5 \mathrm{meV}$, the sweep directions are indicated with arrows. The current becomes zero above $4 \mathrm{~T}$ since the double dot then shifts into a Coulomb blocked state due to the Zeeman shift of $T_{+}(1,1)$. The same device was previously studied in Ref. [19].

beyond 10-20 mT, and the system enters a metastable low current state [10]. At higher fields, both low and high current states are observed, as well as transitions between the two. The transitions are always abrupt for all measured samples, on the $30 \mathrm{~ms}$ time scale of data acquisition.

In Fig. 1(c) we present an example of a hysteretic current trace. We see that the low current state can be "dragged" up to very high fields (over $4 \mathrm{~T}$ ). The reversed sweep shows a distinct high current state down to $\sim 0.7 \mathrm{~T}$, where the current switches. If the magnetic field is fixed to a value inside the hysteretic regime, the double dot may suddenly switch after minutes or seconds, or remain in either of the states for as long as hours (see Supplemental Material [21] for time-dependent measurements). We note that the higher noise observed in the low current state does not represent typical behavior, often the current fluctuations are larger in the high current state.

Electron spin-nuclear spin feedback is known to exhibit complex dynamics, including hysteresis [6,11], multistabilities [22], and fast switching between different stable states [5,23]. This suggests that the observed switching and hysteresis might be due to DNP. Earlier experiments attempted to extract the degree of nuclear polarization directly from the hysteresis curves $[9,10]$. In this interpretation, the maximum nuclear polarization is simply given by the size of the hysteresis loop. In our case, Fig. 1(c) would present a contradiction to such an interpretation. The field range of hysteresis exceeds $3 \mathrm{~T}$, which is a few times larger than the effective field corresponding to full polarization for InAs $(\approx 1.0-1.5 \mathrm{~T}$, depending on the effective electronic $g$ factor). This shows that a straightforward analysis of the hysteresis curves does not provide an estimate for the maximum nuclear fields in the dots.

We are able to determine the nuclear polarization directly, by probing the effective Zeeman splitting of the electrons. To this end, a continuous wave $\mathrm{GHz}$-frequency electric field is applied to one of the gates. The oscillating electric field drives transitions between the Zeeman-split spin-orbital eigenstates of electrons. When the ac frequency matches the Larmor precession frequency $f$ in one of the dots, additional transitions within the $(1,1)$ manifold are induced and extra current flows through the double dot. In the presence of DNP, the Larmor frequency is given by $2 \pi f=\left|g \mu_{B}\left(\vec{B}_{\text {ext }}+\vec{B}_{N}\right)\right|$, where $g$ is the effective $g$ factor in the quantum dot, $\mu_{B}$ is the Bohr magneton, $\vec{B}_{N}$ is the effective nuclear field, and we have set $\hbar=1$ for convenience. Therefore, a finite $\vec{B}_{N}$ will reflect in a shift of $f$.

The EDSR spectroscopy is performed on a second device, the spectrum is shown in Fig. 2(b) and the high and low current states in Fig. 2(a). In the low current state we fit the observed resonance frequency to $2 \pi f=\left|g \mu_{B} B_{\text {ext }}\right|$, yielding $g=8.7 \pm 0.1$. Similar $g$ factors were measured in this device in a regime where hysteresis was not observed [3]. The scenario proposed in Ref. [10], in which DNP compensates the external field fully in the low current state to a nearly zero total Zeeman energy, can thus again be ruled out. We also note that the extent of the low current state is asymmetric with respect to the zero field axis [24]. This effect is further explored in the Supplemental Material [21]. A second important observation is that only a single EDSR is observed in the low current state, while in the high current state we see multiple EDSRs. In strongly coupled quantum dots we do expect multiple resonances corresponding to various transitions within the manifold of the $(0,2)$ and $(1,1)$ singlet and triplet states [13]. The observation of only a single resonance in the low current state is thus surprising. We at least expect to see two EDSR lines due to a $g$-factor difference between the two dots [3].

We also detect a $0.3 \mathrm{GHz}$ shift in the primary resonance frequency at the transition boundary [Fig. 2(b), inset]. This suggests that the two current states do have different nuclear polarizations, the difference being however rather small (at least in one of the dots). How could a small change in polarization alter the current through the double dot by an order of magnitude? And why is only a single EDSR observed in the low current state? In what follows we propose an explanation based on the gradient in the $z$ projection of the nuclear fields $\Delta B_{N}^{z}$ over the two dots [8].

Let us first consider the basics of transport through a spin-blocked $(1,1) \rightarrow(0,2)$ transition in the presence of 


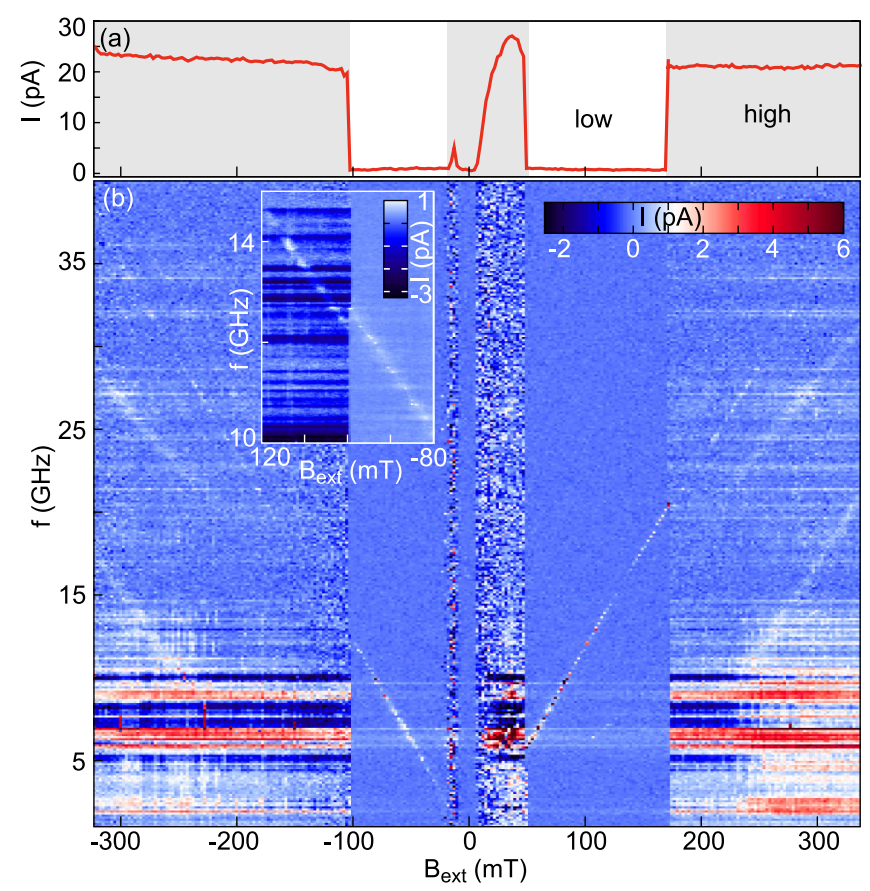

FIG. 2 (color online). (a) Double dot current as a function of magnetic field (device $2, V_{\mathrm{dc}}=7.5 \mathrm{mV}$, and $\varepsilon \approx 2.5 \mathrm{meV}$ ). The gray and white background indicates the high and low current states respectively. (b) The frequency of the ac voltage on a local gate is swept, while the magnetic field is stepped. At each point, we measure $50 \mathrm{~ms}$ with ac excitation on the gate and then $1 \mathrm{~s}$ without. The difference in the two measured currents is plotted. This procedure also avoids dragging of the spin resonance. The inset shows a zoom-in on the spectral line at the transition from the high to low current state. Low ac power is used to minimize switches between metastable states induced by the driving. The horizontal lines at fixed frequency correspond to photon assisted tunneling enhanced by cavity modes in the fridge. This is the same device as studied in Ref. [3].

spin-orbit interaction and in the strong interdot coupling regime. An electron enters the left quantum dot and forms one of the four $(1,1)$ states with an electron on the right dot. Out of the four states, only the $(1,1)$ spin singlet is tunnel coupled to the $(0,2)$ singlet due to spin selection rules [the $(0,2)$ triplet states are at too high energy to play a role]. In the absence of a magnetic field, this results in three blocked states and therefore very low current, proportional to the residual escape rate out of the blocked states. If a finite magnetic field is applied, the spin-orbital $T_{+}(1,1)$ and $T_{-}(1,1)$ are split off by the Zeeman energy $\pm E_{Z}=$ $\pm \bar{g} \mu_{B} B_{\text {ext }}$ ( $\bar{g}$ being the average $g$ factor of the two dots), see Fig. 3(a). Spin-orbit interaction then effectively allows for spin-nonconserving tunneling and couples $T_{ \pm}(1,1)$ to $S(0,2)$, characterized by the energy $t_{\mathrm{SO}}$ [20]. The remaining blocked state $T_{0}(1,1)$ forms the bottleneck for transport and the escape rate out of this state determines the current.

A difference $\Delta E_{Z}$ in effective Zeeman splittings in the two dots mixes $T_{0}(1,1)$ with $S(1,1)$, thereby unblocking the system at finite magnetic field [5], as indicated in
Fig. 3(a). $\Delta E_{Z}$ is contributed to by a difference in the effective $g$ factors of the two dots $\Delta g$, as well as by a nuclear field gradient along the $z$ axis. Since $T_{0}(1,1)$ constitutes the bottleneck in the transport cycle, a change in $\Delta B_{N}^{z}$ (and thus in $\Delta E_{Z}$ ) could indeed have a significant effect on the current. We support this statement by performing transport simulations including a Zeeman gradient. Figure 3(b) shows the calculated double dot current vs $B_{\text {ext }}$, for two values of $\Delta E_{Z}$. We used a rate equation model that includes the effects of hyperfine and spin-orbit interactions [19]. A gradient of only a few mT is sufficient to increase the current by almost an order of magnitude. Note that we have previously reported higherthan-expected current levels in the strong coupling regime [19]. We now propose that this higher current was due to the Zeeman gradient over the two dots which was not included in the model at the time.

In InAs double dots, it has been observed that typically $\Delta g / \bar{g}=1 \%-10 \%[3,14]$. At applied fields of $100 \mathrm{mT}$ this mismatch would induce a Zeeman gradient of several $\mathrm{mT}$ and thus lead to a considerable increase of current due to lifting of the spin blockade [Fig. 3(b)]. Low current could arise when a small nuclear field gradient exactly compensates the Zeeman gradient due to $\Delta g$. The low current state thus has $\Delta E_{Z} \approx 0$. This idea also explains that only a single EDSR is observed in the low-current state [Fig. 2(b)] in contrast to Ref. [3] where in the same device two resonances corresponding to two dots were resolved.

In what follows we show theoretically that electronnuclear spin flip-flops indeed can drive the double dot towards $\Delta E_{Z}=0$ and keep it there. In Fig. 4(a) we plot the spectrum of the $(1,1)$ states as a function of $\Delta E_{Z}$ close to $\Delta E_{Z}=0$. The thickness of the lines in the spectrum corresponds to the occupation probabilities of the four states one finds when taking into account the coupling to the decaying $(0,2)$ singlet: at $\Delta E_{Z}=0$ the system has one (a)

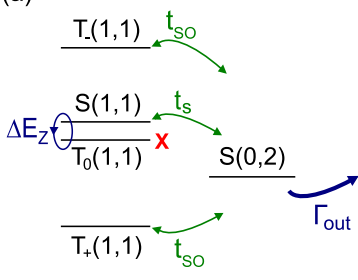

(b)

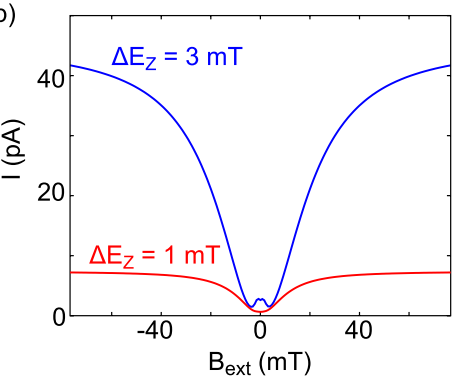

FIG. 3 (color online). (a) Sketch of the spectrum of the five electronic states. The coupling between the different states, as well as the decay rate $\Gamma_{\text {out }}$ of $S(0,2)$ are indicated. (b) Simulated current in the presence of an external Zeeman gradient of $1 \mathrm{mT}$ (red trace) and $3 \mathrm{mT}$ (blue trace). We used $\varepsilon=\Gamma_{\text {out }}=1.5 \mathrm{meV}$, $t_{s}=100 \mu \mathrm{eV}, t_{\mathrm{SO}}=25 \mu \mathrm{eV}$, and $\bar{g}=9$. We averaged over $10^{5}$ random nuclear fields taken from a normal distribution with $\sqrt{\left\langle\left(B_{N}^{x, y, z}\right)^{2}\right\rangle}=B_{N}^{\mathrm{rms}}=1 \mathrm{mT}$ for both dots. 
blocked state $\left|T_{0}\right\rangle$ in which it spends all its time. When $\Delta E_{Z}$ deviates from zero, $\left|T_{0}\right\rangle$ and $|S\rangle$ acquire a spinorbital $\uparrow \downarrow$ and $\downarrow \uparrow$ character and the blockade is lifted. Correspondingly, the double dot current increases as $\Delta E_{Z}$ moves away from zero.

At $\Delta E_{Z}=0$ positive and negative pumping rates are balanced. Hyperfine induced electron-nuclear spin flipflops can cause transitions from the only occupied state $\left|T_{0}\right\rangle$ to $|\uparrow \uparrow\rangle$ and to $|\downarrow\rangle$, with equal probabilities. Since $\left|T_{0}\right\rangle$ is an equal superposition of $|\uparrow \downarrow\rangle$ and $|\downarrow \uparrow\rangle$, these processes will not lead to a net pumping of the nuclear fields.

As soon as $\Delta E_{Z}$ deviates from zero, DNP tries to return the system to $\Delta E_{Z}=0$. For example, when $\Delta E_{Z}$ is positive, the most strongly occupied state $\left|T_{0}\right\rangle$ acquires a $\downarrow \uparrow$ character and transitions starting from $\left|T_{0}\right\rangle$ have a preferred direction for spin flips [25]. This yields a DNP with net rates $R_{L}^{-}$and $R_{R}^{+}$[red dashed arrows in Fig. 4(a)]: the left dot is negatively pumped, the right dot positively.

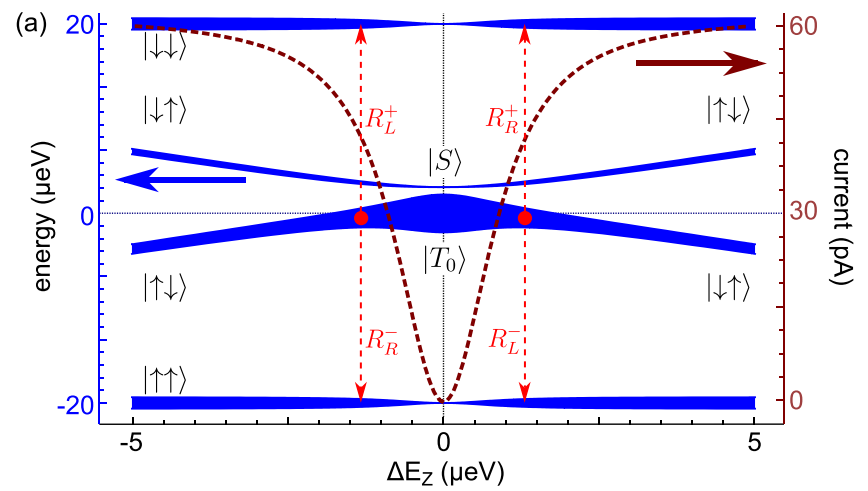

(b)

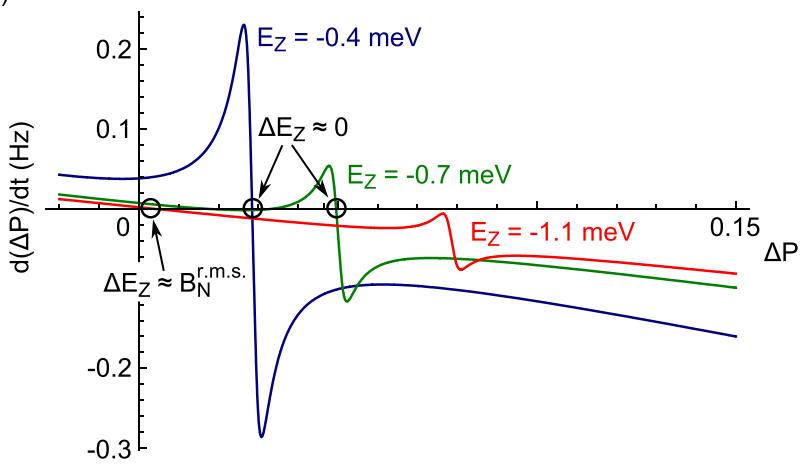

FIG. 4 (color online). (a) Thick blue lines, left scale: Spectrum of the $(1,1)$ states as a function of $\Delta E_{Z}$ for $E_{Z}=-20 \mu \mathrm{eV}, t_{s}=$ $60 \mu \mathrm{eV}, t_{\mathrm{SO}}=40 \mu \mathrm{eV}$, and $\varepsilon=\Gamma_{\text {out }}=1.5 \mathrm{meV}$. The thickness of the lines indicates the occupation probability of the corresponding eigenstate $(4 \mu \mathrm{eV}=1$ ). Brown dashed line, right scale: Current through the double dot as a function of $\Delta E_{Z}$ for the same parameters. Red dashed arrows: Preferred directions of nuclear spin flips close to $\Delta E_{Z}=0$. (b) $d(\Delta P) / d t$ as a function of $\Delta P$ for three different magnetic fields, negative $E_{Z}$ corresponds to a positive field. We used the same parameters as at (a), with $A I=0.7 \mathrm{meV}, I=\frac{1}{2}, N=10^{5}, 1 / \tau=3 \times 10^{-10} \mu \mathrm{eV}$, and $\Delta g / \bar{g}=0.05$. Stable (un)polarized states of the nuclear spin ensembles are indicated.
Both these rates indeed drive the system back to $\Delta E_{Z}=0$. Transitions from $|S\rangle$ have the same asymmetry but with opposite sign and counteract this pumping. However, since the occupation probability of $|S\rangle$ is much smaller than that of $\left|T_{0}\right\rangle$, transitions from $\left|T_{0}\right\rangle$ dominate.

This intuitive picture is confirmed by an explicit calculation of all allowed hyperfine flip-flop rates. The derivation involves a few straightforward steps, following the approach of previous works [6,22] (see the Supplemental Material [21] for details). Assuming nuclear spin 1/2 for simplicity, we calculate each separate transition rate using Fermi's golden rule. Summing over all transition rates, we arrive at an equation of motion for the polarization gradient over the dots:

$$
\frac{d \Delta P}{d t}=-\frac{A^{2}}{4 N^{2} E_{Z}^{2}} \frac{\Gamma_{\text {out }} t_{s}^{2}}{\Gamma_{\text {out }}^{2}+4 \varepsilon^{2}} f(\theta)[\sin 2 \theta+2 \Delta P]-\frac{\Delta P}{\tau},
$$

where $\Delta P=\frac{1}{2}\left(P_{L}-P_{R}\right)$, with the nuclear polarization in left and right dots $-1<P_{L(R)}<1$. $N$ is the number of nuclei in each dot (see Supplemental Material [21] for a discussion of asymmetric dot sizes). $A$ is the average hyperfine coupling energy $(A I \sim 0.7 \mathrm{meV}$ for InAs, with $I$ the average total nuclear spin). The angle $\theta$ is defined by $\tan \theta=\left(\Delta E_{Z}\right)\left(\Gamma_{\text {out }}^{2}+4 \varepsilon^{2}\right) / 2 \varepsilon\left(t_{s}^{2}+t_{\text {SO }}^{2}\right)$, where $\Delta E_{Z}=$ $(\Delta g) \mu_{B} B_{\text {ext }}+A I(\Delta P)$, and the dimensionless function $f(\theta) \sim 1$ is given in the Supplemental Material [21]. In deriving (1), we assumed that $\left|E_{Z}\right| \gg \Gamma_{a}$ for all $(1,1)$ states. We also added a phenomenological nuclear spin relaxation rate $1 / \tau \sim 0.1-1 \mathrm{~Hz}$. The average polarization $P=\frac{1}{2}\left(P_{L}+P_{R}\right)$ is not pumped: DNP merely enhances the relaxation rate of $P$.

In Fig. 4(b) we plot the pumping curve (1) for $\Delta P$ with realistic parameters, for different magnetic fields. We see that at low fields the system has one single stable state close to the low current point $\Delta E_{Z}=0$ (blue curve, $E_{Z}=$ $-0.4 \mathrm{meV})$. At intermediate fields, the high current unpolarized state with $\Delta P \approx 0$ is stable as well (green curve, $\left.E_{Z}=-0.7 \mathrm{meV}\right)$. This bistability can manifest itself in switching and hysteresis. At very high fields, DNP becomes too weak to counteract nuclear spin relaxation and only the high current unpolarized state is stable (red curve, $E_{Z}=-1.1 \mathrm{meV}$ ). Using parameters from Ref. [19] we estimate the maximum field for which DNP can stabilize the $\Delta E_{Z} \approx 0$ to be $6 \mathrm{~T}$, which is indeed consistent with Fig. 1(c) (see Supplemental Material [21] for details).

Often, states of nuclear polarization stabilized by DNP exhibit significantly reduced fluctuations. Using the spinflip rates found from Fermi's golden rule we evaluate the variance of the gradient distribution in the polarized state as compared to the unpolarized state. We find a relative suppression of the mean-square fluctuations of about $\approx 4 \times 10^{-3}$ for the parameters of the blue curve in Fig. $4(\mathrm{~b})$, and of $\approx 9 \times 10^{-3}$ for those of the green curve (see Supplemental Material [21] for details). In the context 
of two-electron single-triplet qubits, this narrowing could lead to an enhancement of the dephasing time $T_{2}^{*}$ by more than an order of magnitude.

We thank M. Rudner, H. Bluhm, E. Rashba, Yu. Nazarov, L. Levitov, L. Vandersypen, and K. Nowack. This work has been supported by NWO/FOM (Netherlands Organization for Scientific Research), by the Alexander von Humboldt Foundation, and through the DARPA program QUEST.

[1] J. R. Petta, A. C. Johnson, J. M. Taylor, E. A. Laird, A. Yacoby, M. D. Lukin, C. M. Marcus, M. P. Hanson, and A. C. Gossard, Science 309, 2180 (2005).

[2] F.H.L. Koppens, K.C. Nowack, and L.M. K. Vandersypen, Phys. Rev. Lett. 100, 236802 (2008).

[3] S. Nadj-Perge, S. M. Frolov, E. P. A. M. Bakkers, and L. P. Kouwenhoven, Nature (London) 468, 1084 (2010).

[4] K. Ono and S. Tarucha, Phys. Rev. Lett. 92, 256803 (2004).

[5] F. H. L. Koppens, J. A. Folk, J. M. Elzerman, R. Hanson, L. H. Willems van Beveren, I. T. Vink, H.P. Tranitz, W. Wegscheider, L.P. Kouwenhoven, and L. M. K. Vandersypen, Science 309, 1346 (2005).

[6] M.S. Rudner and L.S. Levitov, Phys. Rev. Lett. 99, 246602 (2007).

[7] H. O.H. Churchill, A. J. Bestwick, J. W. Harlow, F. Kuemmeth, D. Marcos, C.H. Stwertka, S. K. Watson, and C. M. Marcus, Nat. Phys. 5, 321 (2009).

[8] T. Kobayashi, K. Hitachi, S. Sasaki, and K. Muraki, Phys. Rev. Lett. 107, 216802 (2011).

[9] J. Baugh, Y. Kitamura, K. Ono, and S. Tarucha, Phys. Rev. Lett. 99, 096804 (2007).

[10] A. Pfund, I. Shorubalko, K. Ensslin, and R. Leturcq, Phys. Rev. Lett. 99, 036801 (2007).
[11] I. T. Vink, K. C. Nowack, F. H. L. Koppens, J. Danon, Y. V. Nazarov, and L. M. K. Vandersypen, Nat. Phys. 5, 764 (2009).

[12] L. R. Schreiber, F. R. Braakman, T. Meunier, V. Calado, J. Danon, J. M. Taylor, W. Wegscheider, and L. M. K. Vandersypen, Nat. Commun. 2, 556 (2011).

[13] S. Nadj-Perge, V.S. Pribiag, J. W. G. van den Berg, K. Zuo, S. Plissard, E. P. A. M. Bakkers, S. M. Frolov, and L. P. Kouwenhoven, Phys. Rev. Lett. 108, 166801 (2012).

[14] M. D. Schroer, K. Petersson, M. Jung, and J. Petta, Phys. Rev. Lett. 107, 176811 (2011).

[15] J. M. Taylor, H.-A. Engel, W. Dür, A. Yacoby, C. M. Marcus, P. Zoller, and M. D. Lukin, Nat. Phys. 1, 177 (2005).

[16] S. Foletti, H. Bluhm, D. Mahalu, V. Umansky, and A. Yacoby, Nat. Phys. 5, 903 (2009).

[17] H. Bluhm, S. Foletti, D. Mahalu, V. Umansky, and A. Yacoby, Phys. Rev. Lett. 105, 216803 (2010).

[18] H. Bluhm, S. Foletti, I. Neder, M. Rudner, D. Mahalu, V. Umansky, and A. Yacoby, Nat. Phys. 7, 109 (2011).

[19] S. Nadj-Perge, S. M. Frolov, J. W. W. van Tilburg, J. Danon, Y. V. Nazarov, R. Algra, E. P. A. M. Bakkers, and L. P. Kouwenhoven, Phys. Rev. B 81, 201305(R) (2010).

[20] J. Danon and Y. V. Nazarov, Phys. Rev. B 80, 041301(R) (2009).

[21] See Supplemental Material at http://link.aps.org/ supplemental/10.1103/PhysRevLett.109.236805 for additional data and detailed theory discussion.

[22] J. Danon, I. T. Vink, F. H. L. Koppens, K. C. Nowack, L. M. K. Vandersypen, and Y. V. Nazarov, Phys. Rev. Lett. 103, 046601 (2009).

[23] M.S. Rudner, F. H. L. Koppens, J. A. Folk, L. M. K. Vandersypen, and L.S. Levitov, Phys. Rev. B 84, 075339 (2011).

[24] M.S. Rudner and E. I. Rashba, Phys. Rev. B 83, 073406 (2011).

[25] M. Stopa, J. J. Krich, and A. Yacoby, Phys. Rev. B 81, 041304 (2010). 